\title{
Disruptions within gut microbiota composition induced by improper antibiotics therapy as a probable trigger factor for development of depression - Case Reports
}

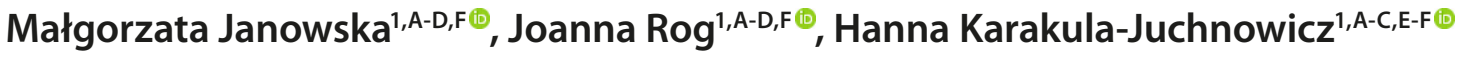 \\ ${ }^{1}$ Ist Department of Psychiatry, Psychotherapy and Early Intervention, Medical University, Lublin, Poland \\ A - Research concept and design, B - Collection and/or assembly of data, C - Data analysis and interpretation, \\ $D$ - Writing the article, E-Critical revision of the article, F- Final approval of article
}

Janowska M, Rog J, Karakula-Juchnowicz H. Disruptions within gut microbiota composition induced by improper antibiotics therapy as a probable trigger factor for development of depression - Case Reports. Ann Agric Environ Med. 2021; 28(4): 713-718. doi: 10.26444/aaem/132452

\section{Abstract}

A growing body of evidence confirms that immune activation and low-grade inflammation could be defined as risks factors for the development and progression of mood episodes. A suggested mechanism leading to immune-inflammatory imbalance is the change in gut microbiota composition provoked by intestinal permeability. Three cases of patients with first episode of depression: a 30-year-old man and two 41 and 46-year-old women are presented in the study. In all cases, the episode was proceeded by the infection of the upper respiratory tract and improper antibiotics therapy. Despite the subsidence of infection, gastrointestinal and depression symptoms appeared. Psychiatric care, anti-depressant treatment, and probiotic supplementation were applied with positive results. Changes in gut microbiota and gut permeability are mechanisms probably involved in the development of mood disorders among the described patients. Lack of microbiota and gut permeability analysis allows defining just a temporary, potential cause-effect relationship between disease symptoms and intestinal microbiome alternations. Further studies to establish the importance of gut bacteria, immune-inflammatory cascade at depression etiopathogenesis and therapy are needed.

\section{Key words}

gut microbiota; major depressive disorder; depression; mood disorder; probiotics; gut microbiome; gut permeability

\section{INTRODUCTION}

Depression is not an isolated disease. During the time of ongoing deepening of knowledge about the inter-relationship between different fields of medicine, affective disorders should also be mentioned in an interdisciplinary way. An increasing number of studies have shown the comorbidity of mental illness with cardiac, thyroid diseases or diabetes [1].

The EZOP Poland study, 'The prevalence of common mental disorders in the population of adult Poles by gender and age structure' conducted between 2008-2011, based on a representative sample of Poles, revealed 3\% of adults between the ages of 18 and 64 years have had as least one episode of depression during their lifetime. Nevertheless, the symptoms of depression are often neglected [2].

Risk factors of affective disorders are quite well known and include biological, somatic, psychosocial, and genetic factors [3]. The factor with a significant effect on immuneinflammation regulation are microorganisms residential to the gastrointestinal tract (GI) - gut microbiota, which are crucial for maintaining health due to the modulation of numerous pathways:

a. they shape both the innate and adaptive immune system throughout life;

b. they activate host immune pathways, trigger the production of inflammatory cytokines, and regulate T-cells function $[4,5]$;

Address for correspondence: Joanna Rog, Ist Department of Psychiatry, Psychotherapy and Early Intervention, Medical University of Lublin, Poland E-mail: joannarog@umlub.pl

Received: 21.07.2020; accepted: 21.11.2020; first published: 22.01.2021 c. they are necessary for proper neurodevelopment [6];

d.they have an effect on the function, regulation, and restoration of tight junctions (TJs) - protein in an apical membrane of blood and brain barrier [7-9]. Lack of integrity in both barriers could provoke GI tract and central nervous system (CNS) abnormalities by passage molecules (also pathogenic and immunogenic) out of the gut to blood circulation and brain [10, 11];

e. microbial digestion and fermentation lead to production metabolites of tryptophan, and derived fatty acids, which have a significant effect on the immune response and brain. Short-chain fatty acids (SCFAs), whose content is a proposed factor of depression phenotype, leading to enhancing immune system functioning, intestinal permeability regulation, and microglia maturation function $[5,12]$.

Studies have shown that the gut microbiota in patients with depression is different from microbiota in healthy controls. In patients with depression, both over- and underrepresentation of some microbiota species are observed, but results are contradictory $[11,13,14]$.

Interpretation of the observed relationship between mood symptoms and gut microbiota should be made very cautiously. Medications taken by MDD patients may have various effects on the composition of the intestinal microbiome. Antidepressive treatment leads to changes in the gut ecosystem, and the shifts are not always colinear to improvement of the patients' mental health Furthermore, somatic disorders commonly experienced by individuals with depression affects the gut ecosystem. Including patients taking drugs 
belonging to a different class influences the results of studies and complicates the drawing of conclusions $[15,16]$.

It is difficult to determine and adequately define a quantitative and qualitative composition of gut microbiota. Some evidence suggests that the composition of microbiota differentiates patients with MDD to a greater extent during the exacerbation period which, therefore, may have a significant impact on disease processes, and the maintenance or the appearance of depressive symptoms [14]. To-date, the results of studies have been unequivocal. Nevertheless, two meta-analyses have shown a potential therapeutic effect of probiotics on mood symptoms, with the results being more promising in patients with the diagnosis of depression [17-20].

\section{OBJECTIVES}

The aim of the study is to present the probable mechanisms for the development of the first depressive episode associated with co-occurring low-grade inflammation, caused by alternations in the gut microbiome and loss of intestinal mucosa integrity as a result of improper probiotic therapy during antibiotic treatment, and non-steroidal anti-inflammatory drug intake.

\section{CASE REPORTS}

Case I. A 30-year-old male, with higher education, living with his wife and 10-year-old son, employed, and practices bodybuilding, had been suffering from hypertension and was taking bisoprolol (1.25 mg/d). Clinical history revealed allergic disease, bronchial asthma and recurrent pharyngitis. Body Mass Index (BMI) $-22.53 \mathrm{~kg} / \mathrm{m}^{2}$. He had taken anabolic steroids (methandienone) several times in the past. The patient's family history revealed that his mother had been treated for depressive disorder, his father had an anxiety disorder. The examination of the patient was his first contact with a psychiatrist.

In January 2018, the patient suffered from an upper respiratory tract infection. The family practitioner advised azithromycin (500 $\mathrm{mg}$ per day (/d) for three days and a probiotic (2 mld CFU Lactobacillus rhamnosus) 3 times/d for 7 days. The symptoms did not subside, and the patient received another antibiotic - clarithromycin $(1000 \mathrm{mg} / \mathrm{d}$ for ten days). During therapy, he did not take probiotics but consumed fermented milk products (yoghurt). His mental state improved, but a slightly raised temperature, cough, and weakness persisted. He again received azithromycin (but under a different trade name) and then cefuroxime $(3 \times 750 \mathrm{mg} / \mathrm{d}$ for 10 days (i.m.)) with good therapeutic effect. Approximately 7 days later, the patient took amoxicillin (oral dose of 1 g every 12 hours for 5 days) due to toothache and swelling of the jaw, with a satisfactory therapeutic effect.

After a 3-week recovery period, symptoms of acute bacterial pharyngitis and tonsillitis occurred and levofloxacin was introduced ( $500 \mathrm{mg} / \mathrm{d}$ for 10 days). Budesonide and ciclesonide (inhalation) were given due to the exacerbation of asthma symptoms. A therapeutic effect was achieved. The patient observed bodyweight reduction - $10 \mathrm{~kg}$ from the time of the first antibacterial treatment. The anthropometric changes were supposedly linked with the occurrence of stomach aches, loose stools, decrease in appetite, and general weakness which included the mental state.
The patient decided to visit the psychiatrist due to mood and psychomotor drive decrease, anhedonia, anxiety, difficulty in thinking ('I am hung up, I can sit for long hours looking straight ahead'), and making decisions, weakness, and fears regarding health. In the last few months before the visit, he experienced panic attacks in the afternoon, which were accompanied by dizziness and chest pains. He complained of head tension without a clear cause. The patient had accurate laboratory diagnostic tests, imaging and microbiological tests performe: morphology, C-reactive protein $(\mathrm{CRP}) \approx 4$, aminotransferases, antistreptolysin-O titer (ASO), thyroid and sex hormones, amylases, ProstateSpecific Antigen (PSA), the magnetic resonance of head, heart echo scan, antibodies to B. burgdorferi according to western blot method). Sertraline $(100 \mathrm{mg} / \mathrm{d})$, trazodone (75 mg/d) and lorazepam (1 mg during the panic attack only) were prescribed during the first psychiatric visit. Multi-strain probiotic supplementation $\left(9 \times 10^{11}\right.$ colonyforming unit (CFU) Streptococcus thermophilus DSM24731 ${ }^{\circ}$, Bifidobacterium longum DSM24736 ${ }^{\circ}$, Bifidobacterium breve DSM24732, Bifidobacterium infantis DSM24737, Lactobacillus acidophilus DSM24735, Lactobacillus plantarum DSM24730, Lactobacillus paracasei DSM24733, Lactobacillus delbrueckii ssp. bulgaricus DSM24734) was also applied.

He also visited various specialist physicians, which allowed the exclusion of a somatic cause. Psychotherapy was suggested. During the second visit to the psychiatrist in June 2018, his mood was significantly improved. He reported periodically occurring low severity anxiety and loose stools. Two months later, his mental state normalized, which was the basis for a gradual reduction in the dose of anti-depressants, full remission, and as a result, discontinuation of treatment.

Case 2. A 41-year-old female with secondary education, currently unemployed, and living with her family and three children. She hds been treated for hypertension (indapamide $1.5 \mathrm{mg} / \mathrm{d}$ ). BMI $-24.34 \mathrm{~kg} / \mathrm{m}^{2}$. There was no psychiatric diagnosis in her family. She associates the onset of mental disorders with bronchitis treated ineffectively with azithromycin (500 mg for three days) and probiotics $\left(6 \times 10^{9}\right.$ CFU Lactobacillus for 7 days) at primary care.

Due to the body temperature rising to $39^{\circ} \mathrm{C}$, she reported to the emergency department, where Amoxycyclin $(1750 \mathrm{mg} / \mathrm{d})$ with clavulanic acid was recommended. The treatment was not successful. During the next visit to a doctor, an antibiogram was carried out, and the patient received penicillin (4 million (mln) international units (IU)/d i.m). This therapy led to the resolution of bronchitis symptoms. However, the woman felt weak and suffered from diarrhoea, leukorrhea, itching, and burning in the genital tract. She also complained about feeling sad, having difficulty thinking, light-headedness, pain and burning in the whole body, and anxiety. The family practitioner recommended the following drugs: antifungal fluconazole (150 mg at one dose), and an antidepressant. Genital candidiasis symptoms disappeared completely. However, the depressive symptoms increased, which were the reason for the visit to the psychiatrist. Loose stools occurred, yet less often than prior to taking fluconazole. The psychiatrist diagnosed a depressive episode and prescribed sertraline $(50 \mathrm{mg} / \mathrm{d}$ in the morning) and trazodone ( $75 \mathrm{mg} / \mathrm{d}$ at night). The patient felt much better, although she was not functioning as previously.

After 7 months of therapy, full remission had not been achieved, another psychiatrist therefore uggested changes 
in the treatment (300 mg quetiapine/d). The patient reported that 'this was better, but everything she did was done with difficulty/heaviness'.

In spring 2017, the patient began therapy at a daily psychiatric ward where she received quetiapine and sertraline. During this time, she became pregnant and the psychiatric treatment was discontinued suddenly at approximately 5 6 week of pregnancy (hbd). At eight hbd, the patient had a miscarriage, after which she took a probiotic $\left(4 \times 10^{9} \mathrm{CFU}\right.$ Lactobacillus rhamnosus) for one month. This resulted in a significant but short-term improvement in her mental state.

However, a drop in her mood again occurred. She reported that her rate of thinking and speech were slowing down and she had significant difficulties in performing daily activities, increased sleep latency, and disruption of the circadian rhythm. She confirmed the presence of resignation and suicidal thoughts without any tendency to realize them. She complained a lot of somatic symptoms, including tension in the head area, burning skin, body pain, muscle stiffness, and periodic loose stools.

She was treated with sertraline $(200 \mathrm{mg} / \mathrm{d})$, quetiapine $(250 \mathrm{mg} / \mathrm{d})$, trazodone $(75 \mathrm{mg} / \mathrm{d})$, combined with probiotic supplementation (multi-graft probiotic, containing 8 strains of probiotic bacteria $5 \times 10^{9}$. (CFU: Bifidobacterium lactis W52, Lactobacillus brevis W63, Lactobacillus casei W56, Lactococcus lactis W19, Lactococcus lactis W58, Lactobacillus acidophilus W37, Bifidobacterium bifidum W23, Lactobacillus salivarius W24 were included). The therapy obtained a decrease in the depressive symptoms.

Case 3. A 46-year-old female, with higher education, working as a teacher in a kindergarden, living with her family and 2 children aged 15 and 16 years, had been treated for hypertension (metoprolol $25 \mathrm{mg} / \mathrm{d}$ ), degenerative disease of the L-S spine, and migraine (which appears after eating high-histamine content or histamine-releasing foods). Clinical history: removal of a non-functional ovarian cyst and occurrence of chronic tonsillitis. BMI $-28.67 \mathrm{~kg} / \mathrm{m}^{2}$. This was her first episode of depression, and neither she nor anyone in her family had previously received psychiatric treatment.

In October 2014, there was an exacerbation of radiculitis of the sacral segment of the spine. At this same time, streptococcal pharyngitis and tonsillitis symptoms e developed. She started antibiotic (cefuroxime $-500 \mathrm{mg} / \mathrm{d}$ per 10 days) and probiotic therapy $\left(2 \times 10^{9} \mathrm{CFU}\right.$ Lactobacillus rhamnosus, 3 times daily per 5 days), simultaneously with meloxicam (nonsteroidal anti-inflammatory drug (NSAID); $7.5 \mathrm{mg} / \mathrm{d}$ ) therapy. After antimicrobial treatment, despite the resolution of streptococcal pharyngitis symptoms, her mental state worsened.

By January 2015, the patient felt sad, tense, had difficulty concentrating, decreased appetite, anhedonia, and difficulty making a decision. The symptoms gradually increased. The depressed mood was accompanied by a feeling of tightness in the throat and stomach, and her psychomotor function was significantly slower.

She decided to visit a psychiatrist and brought her previous laboratory blood tests with her. Their results were within reference values (blood morphology, thyroid-stimulating hormone (TSH), vitamin D, and urine analysis). CRP was slightly increased (3.5). Tianeptine $(12.5 \mathrm{mg} / \mathrm{d})$ and increased physical activity were recommended.
At the next visit in February 2015, there was no improvement in mood symptoms. The ant-depressant was changed to sertraline $(150 \mathrm{mg} / \mathrm{d})$. During the next visit two months later, the patient admitted that since starting the antibiotic therapy she observed loose stools accompanied by a feeling of overflow in the abdomen. These symptoms increased after starting sertraline treatment. The patient's mental state improved slightly - the feeling of fear was less severe.

Due to gastric symptoms, the dose of sertraline was reduced to $100 \mathrm{mg}$, and a multi-strain probiotic was added to the treatment $\left(5 \times 10^{9} \mathrm{CFU}\right.$ : Bifidobacterium lactis W52, Lactobacillus brevis W63, Lactobacillus casei W56, Lactococcus lactis W19, Lactococcus lactis W58, Lactobacillus acidophilus W37, Bifidobacterium bifidum W23, Lactobacillus salivarius W24 with sodium butyrate: $300 \mathrm{mg} / \mathrm{d}$ ).

The patient was also under the care of a dietitian who started dietotherapy, taking into account the likely histamine intolerance and esults of IgG and anti-depressant treatment, the loose stools gradually disappeared, and the patient's psychological well-being completely normalized

\section{DISCUSSION}

The presented case reports of patients with their first depressive episodes indicates a potential causal link between the occurrence of alterations in the gut microbiome composition (as a result of long-term antibiotic use) and worsening of the mental state. Anti-microbial treatment was added without an antibiogram and microbiological diagnostic tests. Simultaneously, the probiotic supplementation was too shortterm. In one case, which could have additionally enhanced changes in gut microbiota occurring after administration of the anti-microbial drugs. The clinical manifestations were stomach aches, bowel movements, loose stools, and genital candidiasis. The anti-bacterial treatment in the described cases reflects the general trend of the widespread over-use of antibiotics.

Analysis of the use of antibiotic in Europe shows Poland is among the 10 European countries with the highest use of antibacterial treatment [21]. As indicated in the described case reports, in order to avoid disruptions in the composition of the intestinal microbiome, accurate diagnostic and verification of the drugs administered earlier are necessary before commencing anti-microbial treatment.

According to studies, depressive symptoms may coexist with changes in the gut microbiota [11, 13, 14, 22, 23]. Bacteria-derived metabolites changes may disrupt the balance of neurotransmitter homeostasis, and intestinal microbial imbalance is linked to excessive gut permeability [24]. This could affect the appearance of various particles translocated to the blood and trigger an immune-inflammatory cascade linked to decreased mood in the patient $[25,26]$. Low-grade inflammation is observed among a quarter of patients with depression [27]. Values of CPR indicating them (>3 mg/l) were observed in all the cases presented cases. The effect of various medications on the microbiome has not been fully elucidated, therefore the potential impact of some of the drugs taken by the patients (e.g. used for hypertension treatment) on the gut environment cannot determine. Taking NSAIDs is associated with distinct microbiota populations, and the bacterial gut composition varied with the type of medication. Using NSAIDs by the described patients 
could have provoked changes in the gut ecosystem [28]. The excessive physical activity described in one of the patients may have negatively impacted on gut function, its structure and its ecosystem. Excessive cortisol secretion, induced by a non-tailored intensity and/or type of physical activity, affects intestinal physiology and morphology [29, 30].

The probable cause for the development of depressive episode in the describes cases could be the direct effect of anti-microbial treatment on CNS. It has been shown that using this type of drug could be linked with the occurrence of encephalopathy, convulsion or Tourette syndrome-like symptoms [31], insomnia, agitation, depression [31, 32], and risk of higher suicidal behaviours [33]. The study by Lurie et al. indicates that one cycle of anti-bacterial treatment is linked to anincreased risk of depression [34].

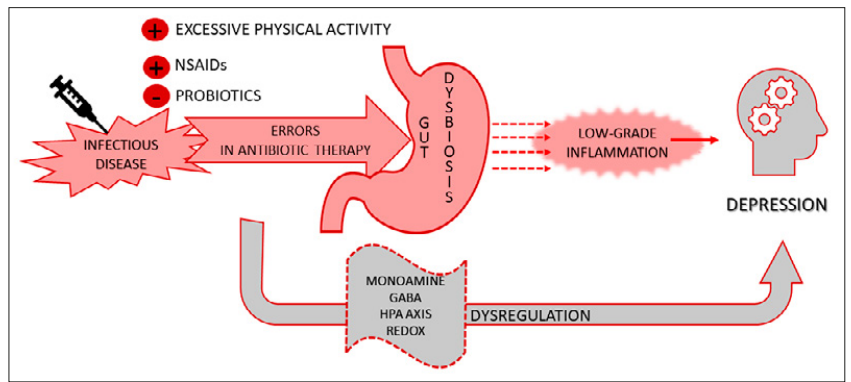

Figure 1. Proposed mechanism connected with gut microbiome alternations, antibiotic therapy, and development of a depressive episode

The mechanisms of antibiotic effect on CNS include:

a. modulation of neurotransmitter levels (amoxicillin and ciprofloxacin) $[35,36]$;

b. GABA receptors antagonism [32];

c. increased oxidation, impaired DNA repair process, and reduction of antioxidant ability (amoxicillin, metronidazole and fluoroquinolones) [37].

As shown above, gut microbiota is medication-treatment dependent. Anti-depressants also have abilities to microbiota modulation although these changes are rather favourable. Anti-microbial potentential, include inhibition of bacterial cell-wall synthesis, anti-plasmid activity, efflux pumps inhibition. SSRIs have a synergistic effect within certain antibiotics and are effective against some bacteria resistant to anti-microbial treatment [38, 39].

Some studies have shown that anti-depressants are able to improve the condition of patients suffering from IBS [40]. Decreased depression severity after anti-depressant treatment may be linked to their a-imicrobial properties, leading to the restoration of gut microbiota balance An unfavourable effect on carbohydrates and lipids metabolism is the weight gain observed during the use of second-generation drugs, to some extent, mediated by microbiome changes [42-44]. Some ant-depressant drugs (i.e. escitalopram have no affect on the gut ecosystem, while others have a favourable action on intestinal microbiota $[45,16]$.

Anti-depressant drugs influencing gut microbiota may be a target for personalized medicine in patients with mood disorders, especially those after anti-biotic treatment. A most promising and known method of restoring gut microbiota balance, is probiotic therapy. Studies confirm the beneficial effect of probiotic supplementation in individuals with symptoms of mood disorders [46]. In physically healthy persons with suicidal thoughts, multi-strain bacteria formulation (B. bifidum, B. lactis, L. acidophilus, L. brevis, L. casei, L. salivarius, L. lactis) reduced rumination [47]. Some strains also have anti-depressant potential in individuals with health-related problems, including diabetes (L. acidophilus, L. casei, B. bifidum, L. fermentum and vitamin D), obese individuals (L. rhamnosus CGMCC1.3724), pregnant women (L. rhamnosus HN001), and patients who have chronic fatigue syndrome (L. casei Shirota) [48-50].

The bacteria strains could improve depressive and stress symptoms, simultaneously with the reduction of HPA axis reactivity $[51,52]$. The beneficial effect of bacteria is related to their ability to reduce the concentration of pro-inflammatory factors [53, and studies aimed at finding specific bacteria strains beneficial for mood disorders are still ongoing [54]. The tailoring of treatment of patients' gut ecosystem creates more promise for depression management and full recovery.

Study limitation. Interpretation of the presented cases must be treated with caution. The occurrence of gut microbiome imbalance was only found according to reported clinical symptoms. No laboratory tests were performed that allowed confirmation of quantity and quality of gut microbial imbalance or increased gut permeability. In the described cases, only time relationship and probable causative relationship between anti-microbial treatment, gut microbiome alternation, and the presence of a depressive episode could be concluded.

\section{CONCLUSIONS}

1. The presented case reports indicate disruptions within the intestinal microbiome composition, caused by improper anti-biotic therapy without adequate probiotic supplementation, as a probable mechanism for the development of the first episode of depression.

2. Analysis of the lack of microbiota and gut permeability allowed defining only a temporary, potential cause-effect relationship between depressive symptoms and intestinal microbiota.

3. Despite proper supplementation of probiotics, using medication from 2 other groups (antibiotics, and NSAIDs) together could lead to the disruption of microbiota composition and function, and increase the risk/promote the occurrence of depressive disorders.

4. During depression episode treatment, it is worth considering the inclusion of psychobiotics, especially in cases with a lack of remission and co-existing gastrointestinal symptoms.

5. Further studies to establish the importance of the intestinal microbiome, immune-inflammatory cascade at depression etiopathogenesis and therapy, are needed.

\section{REFERENCES}

1. Dudek D, Siwek M. Współistnienie chorób somatycznych i depresji. Psychiatria. 2007; 4(1): 17-24.

2. Moskalewicz J, Wciórka J, Stokwiszewski J, Rabczenko D, Kessler RC. The prevalence of common mental disorders in the population of adult Poles by sex and age structure-an EZOP Poland study. Psychiatr Pol. 2015; 49(1): 15-27.

3. Jasik K, Jaślikowska U, Zbrojkiewicz M, et al. Czynniki związane z występowaniem depresji u osób dorosłych. Przegląd systematyczny literatury polskiej w latach 2009-2014 [Factors related to occurrence of 
depressive disorders in adults. A systematic review of Polish literature in years 2009-2014]. Journal of Education, Health and Sport. 2016; 6(4): 297-318

4. Wu H, Tremaroli V, Bäckhed F. Linking microbiota to human diseases: a systems biology perspective. Trends in Endocrinology \& Metabolism. 2015; 26(12): 758-770.

5. Fung TC, Olson CA, Hsiao EY. Interactions between the microbiota, immune and nervous systems in health and disease. Nature neuroscience. $2017 ; 20(2): 145$.

6. Lu J, Lu L, Yu Y, Cluette-Brown J, Martin CR, Claud EC. Effects of intestinal microbiota on brain development in humanized gnotobiotic mice. Scientific reports. 2018; 8(1): 1-16.

7. Nakata K, Sugi Y, Narabayashi H, et al. Commensal microbiota-induced microRNA modulates intestinal epithelial permeability through the small GTPase ARF4. Journal of Biological Chemistry. 2017; 292(37): 15426-15433.

8. Braniste V, Al-Asmakh M, Kowal C, et al. The gut microbiota influences blood-brain barrier permeability in mice. Science translational medicine. 2014; 6(263): 263ra158-263ra158.

9. Kelly JR, Kennedy PJ, Cryan JF, Dinan TG, Clarke G, Hyland NP. Breaking down the barriers: the gut microbiome, intestinal permeability and stress-related psychiatric disorders. Frontiers in cellular neuroscience. 2015; 9: 392.

10. Erickson MA, Dohi K, Banks WA. Neuroinflammation: a common pathway in CNS diseases as mediated at the blood-brain barrier. Neuroimmunomodulation. 2012; 19(2): 121-130.

11. Kelly JR, Clarke G, Cryan JF, Dinan TG. Brain-gut-microbiota axis: challenges for translation in psychiatry. Annals of Epidemiology. 2016; 26(5): 366-372.

12. Skonieczna-Żydecka K, Grochans E, Maciejewska D, et al. Faecal short chain fatty acids profile is changed in Polish depressive women. Nutrients. 2018; 10(12): 1939.

13. Naseribafrouei A, Hestad K, Avershina E, et al. Correlation between the human fecal microbiota and depression. Neurogastroenterology \& Motility. 2014; 26(8): 1155-1162.

14. Jiang $\mathrm{H}$, Ling Z, Zhang $\mathrm{Y}$, et al. Altered fecal microbiota composition in patients with major depressive disorder. Brain, behavior, and immunity. 2015; 48: 186-194.

15. Wu H, Esteve E, Tremaroli V, et al. Metformin alters the gut microbiome of individuals with treatment-naive type 2 diabetes, contributing to the therapeutic effects of the drug. Nature Medicine. 2017; 23(7): 850-858. doi: $10.1038 / \mathrm{nm} .4345$

16. Liśkiewicz P, Pełka-Wysiecka J, Kaczmarczyk M, et al. Fecal Microbiota Analysis in Patients Going through a Depressive Episode during Treatment in a Psychiatric Hospital Setting. Journal of Clinical Medicine. 2019; 8(2): 164. doi:10.3390/jcm8020164

17. Huang R, Wang K, Hu J. Effect of probiotics on depression: a systematic review and meta-analysis of randomized controlled trials. Nutrients. 2016; 8(8): 483.

18. Akkasheh G, Kashani-Poor Z, Tajabadi-Ebrahimi M, et al. Clinical and metabolic response to probiotic administration in patients with major depressive disorder: a randomized, double-blind, placebo-controlled trial. Nutrition. 2016; 32(3): 315-320.

19. Köhler O, Petersen L, Mors O, Gasse C. Inflammation and depression: combined use of selective serotonin reuptake inhibitors and NSAIDs or paracetamol and psychiatric outcomes. Brain and behavior. 2015; 5(8): e00338

20. Ng QX, Peters C, Ho CYX, Lim DY, Yeo W-S. A meta-analysis of the use of probiotics to alleviate depressive symptoms. Journal of affective disorders. 2018; 228: 13-19.

21. Wojkowska-Mach J, Godman B, Glassman A, et al. Antibiotic consumption and antimicrobial resistance in Poland; findings and implications. Antimicrobial Resistance \& Infection Control. 2018; 7(1): 136. doi: 10.1186/s13756-018-0428-8

22. Zheng P, Zeng B, Liu M, et al. The gut microbiome from patients with schizophrenia modulates the glutamate-glutamine-GABA cycle and schizophrenia-relevant behaviors in mice. Sci Adv. 2019; 5(2): eaau8317. doi: $10.1126 /$ sciadv.aau 8317

23. Stevens BR, Goel R, Seungbum K, et al. Increased human intestinal barrier permeability plasma biomarkers zonulin and FABP2 correlated with plasma LPS and altered gut microbiome in anxiety or depression. Gut. 2018; 67(8): 1555-1557.

24. Dinan TG, Cryan JF. Melancholic microbes: a link between gut microbiota and depression? Neurogastroenterology \& Motility. 2013; 25(9): 713-719.

25. Karakula-Juchnowicz H, Gałęcka M, Rog J, et al. The food-specific serum IgG reactivity in major depressive disorder patients, irritable bowel syndrome patients and healthy controls. Nutrients. 2018; 10(5): 548.

26. Karakuła-Juchnowicz H, Pankowicz H, Juchnowicz D. Psychobiotics: new possibilities for treatment of affective disorders? Pharmacotherapy in Psychiatry and Neurology. 2016; (2015 volume 31 issue 3-4): 229-242. doi: 10.17393/fpn.2016.01.005

27. Osimo EF, Baxter LJ, Lewis G, Jones PB, Khandaker GM. Prevalence of low-grade inflammation in depression: a systematic review and metaanalysis of CRP levels. Psychological medicine. 2019; 49(12): 1958-1970.

28. Rogers MA, Aronoff DM. The influence of non-steroidal antiinflammatory drugs on the gut microbiome. Clinical Microbiology and Infection. 2016; 22(2): 178-el.

29. Yuan X, Zhang P, Wang Y, et al. Changes in metabolism and microbiota after 24-week risperidone treatment in drug naïve, normal weight patients with first episode schizophrenia. Schizophrenia Research. 2018; 201: 299-306. doi: 10.1016/j.schres.2018.05.017

30. Exercise-induced stress behavior, gut-microbiota-brain axis and diet: a systematic review for athletes | SpringerLink. Accessed October 21, 2020. https://link.springer.com/article/10.1186/s12970-016-0155-6

31. Rezaei NJ, Bazzazi AM, Alavi SAN. Neurotoxicity of the antibiotics: A comprehensive study. Neurology India. 2018; 66(6): 1732. doi: 10.4103/0028-3886.246258

32. Zareifopoulos N, Panayiotakopoulos G. Neuropsychiatric Effects of Antimicrobial Agents. Clin Drug Investig. 2017; 37(5): 423-437. doi: 10.1007/s40261-017-0498-z

33. Quinolone antibiotics and suicidal behavior: analysis of the World Health Organization's adverse drug reactions database and discussion of potential mechanisms | SpringerLink. Accessed October 21, 2020. https://link.springer.com/article/10.1007/s00213-016-4300-3

34. Lurie I, Yang Y-X, Haynes K, Mamtani R, Boursi B. Antibiotic Exposure and the Risk for Depression, Anxiety, or Psychosis: A Nested CaseControl Study. J Clin Psychiatry. 2015; 76(11): 1552-1528. doi: 10.4088/ JCP. $15 \mathrm{~m} 09961$

35. Evidence for neurotoxicity associated with amoxicillin in juvenile rats - O Atli, U Demir-Ozkay, S Ilgin, TH Aydin, EN Akbulut, E Sener, 2016. Accessed October 21, 2020. https://journals.sagepub.com/doi/ abs/10.1177/0960327115607948

36. Full article: Ciprofloxacin-induced neurotoxicity: evaluation of possible underlying mechanisms. Accessed October 21, 2020. https://www. tandfonline.com/doi/full/10.3109/15376516.2015.1026008

37. Czarny P, Gałecki P, Sliwinski T. Stres oksydacyjny oraz uszkodzenia i naprawa DNA w zaburzeniach depresyjnych Oxidative stress, DNA damage and repair in depression disorders. Published online December 1, 2015. doi: 10.17393/fpn.2016.01.006

38. Kruszewska H, Zareba T, Tyski S. Examination of antimicrobial activity of selected non-antibiotic medicinal preparations. Acta poloniae pharmaceutica. 2012; 69: 1368-1371.

39. Munoz-Bellido JL, Munoz-Criado S, Garcì-Rodrìguez JA. Antimicrobial activity of psychotropic drugs: Selective serotonin reuptake inhibitors. International Journal of Antimicrobial Agents. 2000; 14(3): 177-180. doi: 10.1016/S0924-8579(99)00154-5

40. Effect of Antidepressants and Psychological Therapies in Irr...: Official journal of the American College of Gastroenterology|ACG. Accessed October 21, 2020. https://journals.lww.com/ajg/Abstract/2019/01000/ Effect_of_Antidepressants_and_Psychological.11.aspx

41. Macedo D, Filho AJMC, Soares de Sousa CN, et al. Antidepressants, antimicrobials or both? Gut microbiota dysbiosis in depression and possible implications of the antimicrobial effects of antidepressant drugs for antidepressant effectiveness. Journal of Affective Disorders. 2017; 208: 22-32. doi: 10.1016/j.jad.2016.09.012

42. Skonieczna-Żydecka K, Łoniewski I, Misera A, et al. Second-generation antipsychotics and metabolism alterations: a systematic review of the role of the gut microbiome. Psychopharmacology. 2019; 236(5): 14911512. doi: 10.1007/s00213-018-5102-6

43. Pełka-Wysiecka J, Kaczmarczyk M, Bąba-Kubiś A, et al. Analysis of Gut Microbiota and Their Metabolic Potential in Patients with Schizophrenia Treated with Olanzapine: Results from a Six-Week Observational Prospective Cohort Study. Journal of Clinical Medicine. 2019; 8(10): 1605. doi: 10.3390/jcm8101605

44. Bahr SM, Tyler BC, Wooldridge N, et al. Use of the second-generation antipsychotic, risperidone, and secondary weight gain are associated with an altered gut microbiota in children. Translational Psychiatry. 2015; 5(10): e652-e652. doi: 10.1038/tp.2015.135

45. Liśkiewicz P, Kaczmarczyk M, Misiak B, et al. Analysis of gut microbiota and intestinal integrit markers of inpatients with major depressive disorder. Progress in Neuro-Psychopharmacology and Biological Psychiatry. Published online August 19, 2020: 110076. doi: 10.1016/j.pnpbp.2020.110076 
46. Łoniewski I, Misera A, Skonieczna-Żydecka K, et al. Major Depressive Disorder and gut microbiota - Association not causation. A scoping review. Progress in Neuro-Psychopharmacology and Biological Psychiatry. Published online September 23, 2020: 110111. doi: 10.1016/j. pnpbp.2020.110111

47. Steenbergen L, Sellaro R, van Hemert S, Bosch JA, Colzato LS. A randomized controlled trial to test the effect of multispecies probiotics on cognitive reactivity to sad mood. Brain Behav Immun. 2015; 48: 258-264. doi: 10.1016/j.bbi.2015.04.003

48. Effect of Lactobacillus rhamnosus HN001 in Pregnancy on Postpartum Symptoms of Depression and Anxiety: A Randomised Double-blind Placebo-controlled Trial. Accessed October 21, 2020. https://www.ncbi. nlm.nih.gov/pmc/articles/PMC5652021/

49. Raygan F, Ostadmohammadi V, Bahmani F, Asemi Z. The effects of vitamin D and probiotic co-supplementation on mental health parameters and metabolic status in type 2 diabetic patients with coronary heart disease: A randomized, double-blind, placebocontrolled trial. Prog Neuropsychopharmacol Biol Psychiatry. 2018; 84(Pt A): 50-55. doi: 10.1016/j.pnpbp.2018.02.007

50. Rao AV, Bested AC, Beaulne TM, et al. A randomized, double-blind, placebo-controlled pilot study of a probiotic in emotional symptoms of chronic fatigue syndrome. Gut Pathog. 2009; 1: 6. doi: 10.1186/17574749-1-6

51. Messaoudi M, Violle N, Bisson J-F, Desor D, Javelot H, Rougeot C. Beneficial psychological effects of a probiotic formulation (Lactobacillus helveticus R0052 and Bifidobacterium longum R0175) in healthy human volunteers. Gut Microbes. 2011; 2(4): 256-261. doi: 10.4161/ gmic.2.4.16108

52. Misiak B, Łoniewski I, Marlicz W, et al. The HPA axis dysregulation in severe mental illness: Can we shift the blame to gut microbiota? Progress in Neuro-Psychopharmacology and Biological Psychiatry. 2020; 102: 109951. doi: 10.1016/j.pnpbp.2020.109951

53. Skonieczna-Żydecka K, Marlicz W, Misera A, Koulaouzidis A, Łoniewski I. Microbiome-The Missing Link in the Gut-Brain Axis: Focus on Its Role in Gastrointestinal and Mental Health. Journal of Clinical Medicine. 2018; 7(12): 521. doi: 10.3390/jcm7120521

54. Karakula-Juchnowicz H, Rog J, Juchnowicz D, et al. The study evaluating the effect of probiotic supplementation on the mental status, inflammation, and intestinal barrier in major depressive disorder patients using gluten-free or gluten-containing diet (SANGUT study): a 12-week, randomized, double-blind, and placebo-controlled clinical study protocol. Nutr J. 2019; 18(1): 50. doi: 10.1186/s12937-019-0475-x 